\title{
COVID-19 pneumonitis rehabilitation: when is the best time for a follow-up chest X-ray?
}

\author{
Authors: Carl Hartelius, ${ }^{\mathrm{A}}$ Anita Jayadev ${ }^{\mathrm{A}}$ and Joanne King ${ }^{\mathrm{A}}$
}

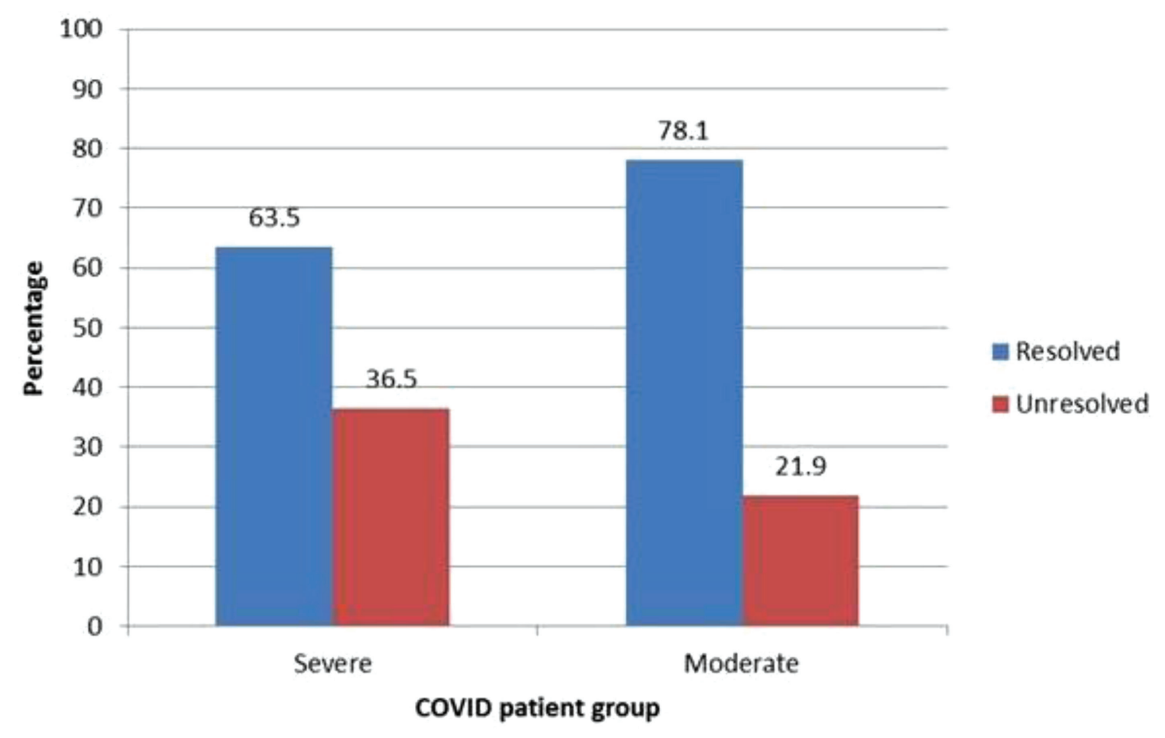

Fig 1. Resolved and unresolved chest $\mathrm{X}$-ray on follow-up in severe and moderate cases of COVID-19.

\section{Introduction}

It has been established that COVID-19 viral pneumonitis is associated with chest X-ray (CXR) changes, predominantly peripheral and basal patchy ground glass opacities. ${ }^{1}$ However, what was less clear was what to expect after recovery from acute illness and the subsequent resolution which made follow-up pathways more difficult to establish. In severe acute respiratory syndrome (SARS) and Middle East respiratory syndrome coronavirus (MERS-CoV) 65\% in radiological follow-up demonstrate complete CXR resolution at a median of 43 days. ${ }^{2,3}$ There were also variable numbers of radiological and clinical features of pulmonary fibrosis up to $60 \%$ but overall case numbers were small. ${ }^{2,3}$ We aimed to establish the proportion of CXR resolution following discharge in patients with COVID-19 pneumonitis and use this information to guide CXR timing in follow up.

\section{Materials and methods}

British Thoracic Society (BTS) guidelines for COVID-19 follow up formed the basis of local guidelines. BTS advised follow up for COVID-19 pneumonitis patients with CXR changes based on stratification into two groups; severe and moderate. The severe group included patients managed in ICU or HDU, who had protracted continued positive pressure ventilation, bi-level non-invasive ventilation or high fractions of inspired oxygen, or with new home oxygen. ${ }^{4}$ BTS advised follow-up at 4-6 weeks for severe cases with a face-to-face review within 12 weeks, while for moderate cases a virtual follow-up within 12 weeks was advised. Both groups were advised to have a CXR within 12 weeks. ${ }^{4}$ Local approach followed this, with the exception of bringing forward CXR for severe patients to 6 weeks. We analysed all follow-up appointments in order to establish CXR resolution and the presence of ongoing symptoms. We also reviewed the local Picture Archiving and Communication System to establish actual timing between abnormal to follow-up CXR.

\section{Results and discussion}

We had 63 patients with severe COVID-19 and 41 patients with moderate COVID-19. We found that in the severe 


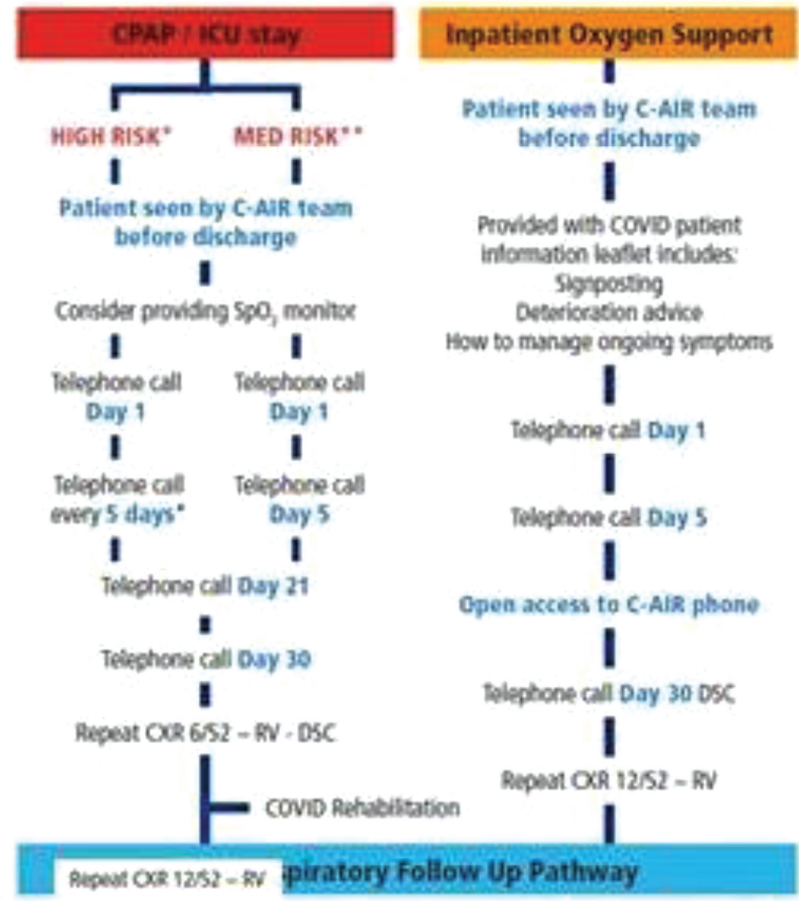

Connhlted te carlinge

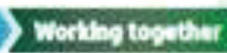

Foding the instiese



If discharged to community bed, Nursing or Residential care for GP / community follow up as required

- At diniciars discoesion

Fig 2. Supportive discharge pathway for patients admitted as COVID-19 positive.

group $63.5 \%$ had resolved CXR compared with $78.1 \%$ in the moderate group (Fig 1). While the severe group were followed up at 6 weeks and the moderate group at 12 weeks, we found that due to the prolonged admissions of the severe group, the CXR timing was similar; 62 days on median for the severe, and 66 for the moderate, making them comparable in terms of timing.

We also found that in the severe group, those with resolved CXR were $65.9 \%$ asymptomatic and $24.4 \%$ symptomatic, those with unresolved CXR were $4.5 \%$ asymptomatic and $72.7 \%$ symptomatic. In the moderate group, those with resolved CXR were $75.0 \%$ asymptomatic and $25.0 \%$ symptomatic, and those with unresolved CXR were $33.3 \%$ asymptomatic and $66.7 \%$ symptomatic.

\section{Conclusion}

We concluded that follow-up CXR at 10 weeks should be recommended for both moderate and severe COVID-19 cases as resolution was over $70 \%$. We hope this will reduce the number of unnecessary CXR while allowing poor resolution to be identified in a timely manner. We now offer both severe and moderate groups 10-week follow-up with a CXR, and COVID-19 Adult Integrated Respiratory (C-AIR) team follow up immediately following discharge (Fig 2).

\section{Conflicts of interest}

None declared.

\section{References}

1 Jin X, Lian JS, Hu JH et al. Epidemiological, clinical and virological characteristics of 74 cases of coronavirus-infected disease 2019 (COVID-19) with gastrointestinal symptoms. Gut 2020;69:1002-9.

2 Hui DS, Joynt GM, Wong KT et al. Impact of severe acute respiratory syndrome (SARS) on pulmonary function, functional capacity and quality of life in a cohort of survivors. Thorax 2005;60:401-9.

3 Das KM, Lee EY, Singh R et al. Follow-up chest radiographic findings in patients with MERS-CoV after recovery. Indian J Radiol Imaging 2017;27:342-9.

4 British Thoracic Society. BTS guidance on respiratory follow up of patients with a clinico-radiological diagnosis of COVID-19 pneumonia. BTS, 2020. www.brit-thoracic.org.uk/about-us/covid-19-information-for-the-respiratory-community [Accessed 03 October 2020]. 